\title{
The Stories We Tell about the Deaths We See
}

Death audit meetings were a much more infrequent but formal accountability mechanism, and one that linked individual deaths and health care workers with their institution, the region, and the rest of the country. The Ministry of Health requires audits of all maternal deaths, as well as neonatal deaths and stillbirths, though the latter two audits never occurred while I was at Mawingu. Thus I concentrate here on the maternal death audit meetings to examine not only documentation and data or accountability but also the discourse that took shape in the discussions about women's deaths that resulted from the audit and the roles these had in shaping responsibility for maternal deaths. Maternal death audit meetings became a bureaucratic technology and tool of reproductive governance that, in opposition to their planned purpose, worked to normalize poor reporting and the number of deaths that occurred in the region.

I first observed a maternal death audit meeting in 2013 while conducting a pilot study at Mawingu. At that time, I still had not decided to study maternal mortality, but the discussion and the information presented at the meeting piqued my interest and contributed to the ultimate direction of my work. In this initial audit meeting, the participants determined that four of the six deaths under review would have been avoidable if the hospital had more efficiently managed the woman's condition or had taken a more comprehensive patient history. It was clear from the discussion that bureaucratic mishaps, systemic malfunctions, and social relations contributed to these lapses in care provision and that while drugs and procedures might be vital for ensuring a woman's survival, simply reaching a hospital (by overcoming sometimes considerable barriers) did not guarantee lifesaving help.

In these meetings, the discussion of a case always ended with the same question: Was this death preventable? ${ }^{2}$ Nine times out of ten, the answer was yes, this death was preventable. What varied vastly between deaths was how the death 
occurred, what transpired on the woman's slow or rapid road to death, and who was, ultimately, responsible for ensuring that such a death would not happen again in the future. Though maternal deaths in Rukwa, in Tanzania, and many parts of the world, have declined since the 1990s, globally nearly three hundred thousand women still die each year during and following pregnancy and childbirth, most in low-resource settings, and most from just a small handful of causes. In Tanzania, the Ministry of Health and Social Welfare issued standardized guidelines for facility-based maternal and perinatal death reviews in 2006, though these had been taking place in some facilities from as early as $1984 .^{2}$

During a maternal death audit meeting, the designated health care providers and administrators went over the details of women who had died from pregnancyrelated causes. The usual participants at Mawingu included the regional medical officer (RMO), the medical officer in charge, the maternity nurse and doctor in charge, all of the district medical officers, the regional and district reproductive and child health coordinators (RRCHCOs and DRCHCOs), representatives from various hospital departments including the lab, and maternity nurses. A WHO publication describes the maternal death audit in this way: "A maternal death audit is an in-depth, systematic review of maternal deaths to delineate their underlying health, social, and other contributory factors, and the lessons learned from such an audit are used in making recommendations to prevent similar future deaths. It is not a process for apportioning blame or shame but exists to identify and learn lessons from the remediable factors that might save the lives of more mothers in the future." ${ }^{3}$ And the WHO suggests, building on its text on maternal death audits from 2004:

[Maternal] deaths should be routinely reviewed or audited as an integral aspect of healthcare quality improvement. ... This approach not only takes advantage of innovations in statistics reporting, but simultaneously improves response mechanisms to avoid future deaths. Over the past years, many low-income countries have introduced action-oriented review mechanisms, described under various names including maternal death enquiry, review, or audit. These require analysis of the circumstances of each death, identification of avoidable factors and action to improve care at all levels of the health system, from home to hospital. Much of the responsibility for follow-up actions lies with district and local health authorities. ${ }^{4}$

While officials and clinicians can employ this process in any setting, in any country, it is particularly useful in countries that lack reliable vital statistics and civil registry systems or where a lack of resources may make it difficult to accurately diagnose the cause of death in a more immediate way. During the audit meeting, the participants also discuss potentially contributing factors extending from the woman's family and community or the referring health facility. These discussions enable district health administrators to analyze how they can improve the referral system, infrastructure, and communication in the future in order to save more 
lives. At the end of the discussion of each woman's case, the group agrees on an "action plan" to be carried out in the ensuing months to address the preventable aspects of the woman's death. These meetings play an important role in collecting data, legitimating state efforts to reduce maternal deaths, and demonstrating the efficacy of individual health care institutions.

Since 2013, I have participated in or been present at a total of four such meetings. While Tanzanian Ministry of Health guidelines, as administrators and providers at Mawingu Regional Hospital described them to me, suggest that hospitals or regional health administrations hold these meetings on a quarterly basis, in practice the meetings occurred much less frequently. Sometimes the regional hospital would go seven months or more without convening such a meeting. By the time the maternity ward doctor in charge had called the meeting and notified all the appropriate district and regional-level administrators, the details of each woman's case were long forgotten, turned into an indistinct blur by the passing of time. No two women's deaths followed the same trajectory, making each case unique but with all-too-common underlying similarities. It was these commonalities that the death audit system was designed to pick up and turn into action plans and points of intervention. ${ }^{5}$ In this way, no death is in vain; each woman leaves behind lessons that can be carried forward to prevent the death of another.

In an era of audit and accountability, of counting and an obsession with metrics as the next global health panacea, the maternal death audit meeting holds a new and loftier role as a way to track these deaths, count them, enumerate the "true" extent of the problem that is maternal death, and collect data on the ongoing causes of these deaths. ${ }^{6}$ Policy makers, governments, and global health practitioners contend that these data, by extension, provide them with the keys to reducing or eliminating such deaths. While the numbers and the forms are meant to strip the dead women's lives down to their clinically important constituent parts, these tools of audit culture are in no way value free, no matter how much their inventors might wish this to be so. ${ }^{7}$

Indicators, such as a country's maternal mortality ratio, are key instruments of governance: "Indicators influence governance when they form the basis for political decision making, public awareness, and the terms in which problems are conceptualized and solutions imagined. Conversely, the kinds of information embodied in indicators, the forms in which they are produced and disseminated, and how they function as knowledge are all influenced by governance practices. The production of indicators is itself a political process, shaped by the power to categorize, count, analyze, and promote a system of knowledge that has effects beyond the producers." ${ }^{8}$ In this context, the audit meetings and their attendant paperwork became a technology specifically of reproductive governance, which the state often enforces through the production and deployment of demographic statistics such as those tracking birthrates and maternal death. ${ }^{9}$ Lynn Morgan and Elizabeth Roberts define reproductive governance as "the mechanisms through 
which different historical configurations of actors-such as state institutions, churches, donor agencies, and NGOs-use legislative controls, economic inducements, moral injunctions, direct coercion, and ethical incitements to produce, monitor and control reproductive behaviors and practices." ${ }^{10}$ Often women are the targets of mechanisms of reproductive governance, ${ }^{11}$ but in death audit processes this complex comes to bear forcefully on administrators and clinicians. The nurses, doctors, and health administrators are at once targets of reproductive governance and tools of these regimes as they also work to shape women into responsible reproducers through their interactions with them on the ward on a daily basis. The data they produce in these meetings include vast quantities of information not just about women but about the data's producers themselves and the health system in which they work through what is both present and absent; in producing these reports, the administrators themselves become targets of reproductive governance. In this instance, these parties are responsible for facilitating society's social and biological reproduction through preventing the deaths of pregnant women. Meeting participants must carefully negotiate how much to reveal about their own culpability while also meeting bureaucratic requirements, which if unmet would trigger other sanctions on the hospital and its leaders.

Much like the partograph, a document-cum-technology whose in vivo form is reconstructed for every new use to which nurses and doctors put it, the numbers and checkboxes of maternal death audit meetings cannot be divorced from the values, ethics, and social and institutional powers that brought them into being in the first place and continue to shape their uses. These death audit papers are meant to standardize, but in fact what they do is strip down, reducing complex lives to "yes" or "no" answers, stuffing bodies-mothers and babies-into checkboxes that cannot possibly contain the messiness and conflicting narratives of lives lived and lost, narratives that employ different lenses, take different perspectives, and arise within diverse contexts. Bureaucratic documents, and development projects generally, often seek to "render technical" complex problems that will then be amenable to technical interventions and solutions. ${ }^{12}$ As Tania Li observes, "To render a set of processes technical and improvable an arena of intervention must be bounded, mapped, characterized, and documented; the relevant forces and relations must be identified; and a narrative must be devised connecting the proposed intervention to the problem it will solve"; ${ }^{13}$ maternal death audits and their forms seek to do just that. Yet the vast complexity of people's lives and care practices can never be fully encompassed in these documents, whose very purpose is to reduce, distill, and standardize so that the lessons from them can be presented in terms of interventions and solutions. Importantly, the forms should also comply with global notions of causes of maternal death. Lost here, too, in these forms are the true underlying causes of maternal death, including Tanzania's political economy and other drivers of stratified reproduction; the antipolitics machine is hard at work containing meaningful challenges to the status quo. ${ }^{14}$ 
When I read Form B, the second form of a two-form set sent to the Ministry of Health after each audit meeting, I could discern almost nothing about the course of the woman's illness, how she came to be at the hospital, the context in which she had lived, and, ultimately, anything about her interactions with the government hospital other than her diagnosis. In Tanzania, despite an emphasis on a qualitative, in-depth analysis of each death, the Ministry of Health forms do not provide much space for elaboration of details; "The structured reporting forms . . . are designed to collect mostly medical causes of death and as such are less suitable to guide the team through an analytical discourse on the gaps in service provision, nor stimulate action-oriented dialogue in the forum." ${ }^{15}$ This lack of action-oriented dialogue significantly reduces the impact of the meetings, solidifying them as performative rather than functional.

Typically, the meeting started with opening remarks from the regional medical officer. Then the maternity ward doctor in charge would begin going through the case files. He read through each woman's medical record from beginning to end, pausing for questions or comments on her diagnosis or course of treatment as others present in the meeting asked for clarification. People would point out delays in the care, question the quality of history taking, or ask about the events that had preceded the woman's arrival at the hospital. The first cases received more careful consideration and discussion, with the district-level administrators interjecting comments about a facility lower down the referral chain, or about how we might determine if the woman had received adequate prenatal care. However, I found, particularly as the meetings dragged on for many hours, the attendees began to focus more and more on simply filling in blanks on the form. They began to copy action plans from one woman's form to the next by rote, without any commensurate discussion of the plan's appropriateness. This was also because the meeting's attendees identified similar problems in many of the cases. The increasingly cursory discussion ignored the fact that, in this setting, even the diagnosis is not the thing of certainty it is meant to be. Without much of the necessary diagnostic equipment, and in the absence of a pathologist, even these determinations, sometimes presented as facts, were often merely interpretations based on experience, gut, and best guesses from how a woman's illness had presented itself, what little information could be gleaned from accompanying relatives, and what the woman herself had said before her death.

The Tanzanian Ministry of Health audit guidelines include very little description of the actual review meeting, focusing much more extensively on "hierarchical reporting structure, technical committees, and administrative management of the data." ${ }^{16}$ The metrics of global health have come to have paramount importance, clearly visible in the maternal death reporting structures, overshadowing or precluding more concerted consideration of the complexity of the lives of individuals and even institutions. ${ }^{17}$ Likewise, the discussions about responsibility and clinical decision making, action plans, and outcome indicators in the audit meetings were 
never able to tell the whole story. But, then again, telling the whole story was not the purpose of these meetings; meeting participants distorted the truth for themselves and most certainly for the consumption of higher authorities.

\section{REPORTING AND DATA COLLECTION}

I had a chance to observe the national reporting structures once in January 2015 when I was in Dar es Salaam. In May 2014 I had met a woman working in the Ministry of Health's Reproductive and Child Health Section headquarters in Dar. In January, I visited her office and we discussed her work responsibilities. At that particular time, her boss was away and she was in charge of compiling the weekly reports based on data coming from the regions.

As I was sitting in her office, her computer pinged and she opened an email with the data from the entire country. There were two deaths for the Rukwa region, and under "location" it said Sumbawanga. I asked her if there was any other information about these deaths because I had just come from Sumbawanga and would have been present during the time period the report covered. She speculated the deaths had occurred at Mawingu, but I knew of no deaths that had occurred at the hospital during that same period. While the deaths under the heading of Sumbawanga could have occurred elsewhere in the municipal district, I told her those data made me uncertain about the rest of the information she had been getting. She told me she would call the Rukwa RRCHCO to clarify the details of the deaths. This was a particularly clear demonstration of the uncertainty inherent in these reporting structures. If I had not been present to ask about the data because of my experiences in the region in question, she would not have made a follow-up phone call and the deaths could have been misattributed to the regional hospital or to nowhere in particular at all.

Reporting requirements were routinely a challenge for many of the health care facilities in Rukwa. Some of the doctors at the regional hospital told me they were unsure about how to properly fill out the MTUHA (Mfumo wa Taarifa za Uendeshaji wa Huduma za Afya, Health Management Information System) books for the end-of-month reports for their wards. The hospital often submitted reports late to the regional or ministry levels, and this was a major area the hospital was targeting for improvement during my time there. Maternal death audit meetings often opened with the RMO reminding the district administrators to submit their reports of deaths in a timely fashion.

At the meeting held in May 2015, the RMO said that everyone was to report the number of maternal deaths every week to the ministry and the RMO's office. ${ }^{18} \mathrm{He}$ said it was very important to be following these reporting guidelines because it was an order that came directly from the president himself in 2014. Even if the districts reported zero, the ministry would be satisfied. After this proclamation, two of the DRCHCOs admitted they had not yet turned in all of their data; one said she had 
been late to the meeting because she was trying to find the relevant information from her office.

The RMO went on to draw attention specifically to the Sumbawanga Rural District (Sumbawanga DC) for its consistently late reporting, which had been a problem for more than a year. Highlighting the important role these data could play, he said, "In 2014, when the regional commissioner was in front of Parliament, she sent a request back to me, asking for the number of maternal deaths in the region. I told her that the maternal mortality ratio was 116 per 100,00o. Later, when I finally received the late data from Sumbawanga DC, I had to tell the regional commissioner that actually the real number for the year was 142 . This was even an increase over the previous year's rate of 139 or 138 per 100,00o!" The RMO then pointedly asked the Sumbawanga DC representative at the meeting, "Do you think the regional commissioner would be understanding if we continued to provide her with bad data to use in front of Parliament and the president? You know, that last time, with that late data, the regional commissioner had to admit our region had not seen any reductions in maternal mortality despite even the passing of another year!" Not only did the RMO consider Sumbawanga DC's tardiness to be problematic and disrespectful, but he saw it as a threat to the region's reputation on a national stage. By providing the regional commissioner with data of dubious veracity, the RMO was also threatening the commissioner's credibility publicly and in front of her superiors.

Reporting could go wrong in any number of ways. In some instances I became confused about how the hospital was counting deaths and who was supposed to take responsibility for which deaths. In theory, any woman who had died at the hospital was supposed to be counted and documented as a hospital death. This sounded, to me, like a fairly black-and-white system. Either she was alive when she arrived, or she was not. However, in practice, there was a much less distinct line. The hospital did not want to appear to be making no progress in improving care and reducing deaths, so they were often selective about how they counted the deaths. This selective counting was an effort to shift responsibility onto other sources and away from the hospital. When a woman arrived at the hospital "already dead," it became less important to remember the details of her case, to record her passing, or to track the life of her statistics after her death. The hospital workers and administrators were happy to absolve themselves of deaths that were not theirs. In this case, the ethical negotiations of care and work were straightforward; she was clearly not their problem.

In mid-2014, I was away from the hospital for about six weeks. At the beginning of July, I tried to follow up and collect information on the maternal deaths that had occurred while I was gone. I had heard there had been two or three deaths. I asked Dr. Charles first, and he said that if the deaths hadn't occurred in the maternity ward, if the women hadn't been technically admitted to the ward already, then the deaths would be recorded in the Outpatient Department (OPD), not maternity. 
He and I agreed that it was hard to follow up with deaths in OPD because of their high patient load. The doctors saw so many patients that it could be difficult to differentiate in memory or to remember the specifics of a particular case and how they had dealt with it bureaucratically after they had addressed the immediate health issue. Dr. Charles told me, "Maybe a good place to start would be to go to supervision and see if they have a record because they usually write down all the deaths that occur." I wanted to know then who was responsible for discussing the death and following up, doing the death review. He said, "It depends on where the woman came from, but the districts are supposed to do death reviews to follow up too."

I went to the nursing supervision office and found the supervisor; she told me to ask another nurse in charge of data collection. That nurse then said we should go together to the OPD to try to follow up. We went to ask Dr. Salome, one of the main OPD doctors, who told me she remembered one case from April; the woman had arrived from the village, had been treated for pregnancy-induced hypertension, and had gone home to stay with relatives in Edeni, in town. The woman had then developed further problems, relatives had brought her back to the hospital, and she had died on the way to the hospital. Dr. Salome had verified the death, dead on arrival; she said she had written it on a piece of paper and sent the body to the mortuary. Technically, then, Dr. Salome explained to me, the death didn't occur at the hospital and the death report was sent to the district from which the woman came, to be counted in the records there.

Dr. Salome continued, "I don't remember any death like that from June or the end of May. But maybe it was another doctor here who received her? You know, it can be hard sometimes here. We don't have the MTUHA book for deaths here in OPD." The data nurse, Lulu, nodded and interjected, “These OPD deaths are really difficult to trace. If you knew the dates, we could look at the roster to see who was working there and find them to ask more." But I did not know the dates and was working only on hearsay. I asked, "So then what am I supposed to do?" She told me I should go back to the maternity ward to check. "But," I said, "they didn't reach maternity to be admitted, so where are these deaths counted? Deaths $z a$ wapi? [Deaths of where?]" All the doctors in the OPD office at that moment murmured in agreement that it was an OPD death but that it would be difficult to find even the woman's name. Turning back to Nurse Lulu, who had accompanied me from the supervisor's office, I asked, "What about a report from supervision?" She told me that often those reports were given orally if the person had not been admitted to the hospital, so there probably would not be any further documentation on the part of supervision. And with that, she told me, "Why don't you go back to maternity and make sure there is no documentation there? I think you will find something there. Here, in my office, we haven't yet gotten the data from June because the month just finished." I returned to the maternity ward, to the start of my search, with no further information about these two deaths. 
Back in the ward, I was ranting to Nurse Gire out of frustration about the problem of tracing the deaths. She told me, "I was here for the one who arrived here and almost immediately died. It was eclampsia, I think." I told Gire that Dr. Charles had said, "That woman who came here, she came and was already gasp, gasping, and then she died after only a very short time." Gire responded, "You know, the way we do this, if the woman isn't included in our admission book here, then perhaps it wasn't recorded as a death here. If she was in the book, then it's required that her death be recorded here. If she dies in OPD, then the information, her files, and everything, go back to the district she came from. They are supposed to follow up there. You see?"

After this explanation, Gire went on to tell me about when she had been a reproductive and child health coordinator some years ago: "At that time I started a form to collect better data at the village level about maternal and neonatal deaths. Really, I got better information, and I told them to bring the forms to me every first of the month for the previous month." With pride, she continued, "I got much higher numbers than others were getting, and I told the RMO at the time that this was a severe problem that needed to be addressed. But now, I don't think those forms are still being used. The data that are being reported nowadays are certainly not accurate!" Getting worked up, she said, "I don't believe that deaths have been reduced. and even the number for Tanzania seems unbelievable! We see here in this hospital! I do this work related to maternal mortality from my heart [kutoka moyoni], but it is hard, and it makes your heart heavy."

Gire then asked me if we had done the maternal death audit meeting yet this year. I said, "No! We have still not yet done it, since last year in October or November! We probably have twenty cases to discuss-we can't finish them in one day anymore! Now we have the ones from the end of 2013 in addition to those from this year." Shaking her head, she said, "We need to see if the RMO is around. He should push for the meeting from the top so it happens within the next week, to bring some pressure so it finally happens. The longer we wait, the more details of the cases have been forgotten!" I agreed and said, "It seems to me then it's not very useful for us in order to improve care if no one remembers the details of the case." Gire nodded her assent as another woman walked through the labor room door carrying her basin filled with her belongings. I moved away, leaving Gire to work as I went in search of the ward nurse in charge, Kinaya.

"Samahani Nurse Kinaya! Excuse me! Do you know about those deaths that occurred here in May and June? Where can I find information on what happened or who these women were?" In her brusque, matter-of-fact manner she said, “The medical officer in charge knows about them. He's the one who said not to document them because tunaongeza vifo siyo vya kwetu [we are increasing deaths that are not ours], it looks like we've killed them, but they came already in a bad condition!" Making a mental note to ask Dr. Joseph, I thanked her and moved on. 
That day I did not receive information about the women's names, their home villages, or any other details that would have made it possible for me to follow up. When I asked Dr. Joseph, he simply explained that they kept no records of such deaths at the hospital because the women had not been alive long enough after arriving to actually be officially admitted. He, and others at the hospital with whom I spoke did not feel that the hospital had any responsibility to count these types of deaths of women who arrived "already dead," either literally or figuratively-almost dead-in the case of those who expired shortly after arriving. I asked him if there was even any record of the names of the women, and he told me no. As the system currently functioned, it was feasible that their names would be impossible to find again, and their deaths might go unrecorded at any level. This experience was another instance that caused me, and others such as Nurse Gire, to be suspicious of any reported declines in maternal death at the hospital, in the region, or in Tanzania more generally. This narrative that the women had arrived "already dead" surfaced over and over again in maternal death audit meetings, but even more in the narratives the nurses and doctors constructed when they gave me a more individual explanation of the phenomenon of maternal death in the regional hospital.

These accounting moves and the discourse of women arriving "already dead" served to shift responsibility and accountability outside the hospital. In this way, the providers and administrators were, in an ethical and state-sanctioned manner, able to shrug off these deaths and leave others to worry about their implications. There was no need to increase the work, the numbers, or the emotional burden at the hospital by reporting deaths that had come from elsewhere. In not entering into a relationship with these women as patients or as statistics, the hospital was also refusing them as seekers and recipients of care. The hospital was severing, arguably before the tenuous claim was ever formalized in admission books and logs, the care connection that would have obliged the nurses, doctors, and administrators to take on a dying woman and, as a result, the statistic of her death. Here, they could hide behind seemingly formalized bureaucratic rules about who constituted an admitted patient of the hospital and, bolstered by the bureaucracy, ethically turn down care for the woman. Denying this care relationship was a way of enacting other care relationships, including care for the maternity ward, care for the hospital, and care for the region's reputation on the national level.

\section{AUDIT MEETING ACTORS AND PROCEEDINGS}

In the Rukwa region, maternal death audit meetings were the responsibility of the regional hospital. I asked more than once if the districts, three rural and one urban, also were supposed to hold such meetings to discuss the deaths in their settings, those that had occurred in district-level health facilities or in the community, but 
I never received a clear answer. The only aspect of the protocol that was readily apparent was the role of the regional hospital. The maternity ward doctor in charge was responsible for calling the meeting. With the support of the regional medical officer's office, he sent letters to all of the district medical officers (DMOs), DRCHCOs, the RRCHCO, and the relevant regional hospital staff members. The regional hospital was generally represented by the medical officer in charge, the maternity ward doctor and nurse in charge, a rotating selection of approximately three maternity ward staff nurses, the other physicians assigned to maternity, and, occasionally, a representative from the pharmacy or the laboratory.

This mixture of administrators at various levels, together with regional hospital staff, led to complicated dynamics and subtle power plays. Directly or indirectly, the regional officials blamed the district health administrators for failing to control or properly supervise the health care workers in the many lower-level facilities within their purview. The district officials had little direct control over these subordinates spread throughout many dispensaries and health centers covering huge land areas. This was in contrast to the much more immediate, direct effects the regional officials could have on the regional hospital. The result was that the district officials often became defensive as they had to listen to action plan after action plan in which they were the chief responsible party and the lowest providers in the region, working in the most peripheral dispensaries, the ones meant to be radically changing their practice to prevent these deaths. The district officials barely had the resources to visit their facilities, lacking funds for gasoline or working vehicles that could traverse the region's rugged terrain. Even if they were able to reach facilities, if they regularly visited all of them, the administrators would rarely have time for any other tasks, so great would the time requirements be.

In one audit late in my stay we were discussing action plans and ways to determine if women were getting enough education during antenatal clinic visits. I, drawing on my expertise as a researcher, which the RMO and medical officer in charge had encouraged, pointed out that simply observing dispensary providers during health counseling talks was not a valid way to measure what women were learning at the antenatal clinic. Naturally, I argued, the provider being observed would change his or her behavior on the day of the visit to appear as favorably as possible. One of the DRCHCOs became incensed and accused me of not understanding what it was like in villages and their dispensaries. She told me, "Women lie. They tell you that they didn't receive any counseling to go to the hospital, especially if they are primigravida, but they did! If you ask those providers there, they will tell you they are counseling women, but those women are still starting at home with the TBAs [traditional birth attendants]!" I politely returned that I had, in fact, spent a great deal of time in villages and, specifically, in dispensaries in two different regions of Tanzania. I suggested it might be more appropriate to interview some women at their homes or near the dispensary to see what they were retaining from clinic visits. I also suggested that if providers said they were counseling 
women about their birth plans, but women were still preferring to start out with the TBAs, we should probably take that as an indication that the counseling was inadequate, inappropriate, or unsuited for the women in question. The DRCHCO continued to bluster that I did not know what I was talking about.

After the meeting, Dr. Deo told me that the DRCHCO had gotten so upset because she knew I was right and that she did not want to make more work for herself. I said I should perhaps just not say anything again in the meetings. Dr. Deo looked at me and said, "No, you must keep telling them these things! One day they will have to listen!" My more critical reflections and suggestions, though perhaps empirically sound, were unwelcome because I had upset a balance in which meeting participants complied with documentation requirements and an official technologization of maternal mortality while escaping additional work and new routines, which this resource-poor region would have been unable to adequately support. My foreignness made it easy for the participants to suggest I was unaware of the implications of what I was suggesting, as indeed at the time I was, but not in the ways I had perceived them to be accusing me of being unaware. I was, naively, fixated on suggesting methods or action plan steps that might actually have produced valid outcome indicators, but that was not, in fact, the intent of these meetings. I was operating on an entirely different plane, one likely characterized by a privileged positionality derived from growing up in a world in which I could control many outcomes and in which validity, accuracy, and efficiency were paramount goals. In short, I had developed my subjectivity in a landscape very similar to that which had produced the idea of maternal death audits as technical interventions. I was not from the landscape that actually produced most of the world's maternal deaths, so the important performative aspects of these meetings were somewhat lost on me at the time; unknowingly, I had broken the fourth wall when I had tried to offer what I thought were useful improvements to action plans. I had also undermined the DRCHCO, already in a less privileged position in this meeting of district and regional authorities, her bosses. Bregje De Kok et al. write that for maternal death audits to be most successful (i.e., to change practices), they ought to take place in an environment supporting self-reflection, constructive criticism, and egalitarianism, but local institutions and cultures often do not, or cannot, fully operate in this ideal fashion. ${ }^{19}$ This assessment, however, is predicated on a particular idea of what makes an audit successful. At Mawingu, changed practices were not necessarily the ultimate goal of these meetings, more because of systemic constraints than because of any real lack of will, but they could be successful in other ways.

Despite the inclusion of hospital outsiders in the meeting, representatives from the OPD and the hospital lab were notably absent in most of these meetings. For those deaths that occurred at night, if the maternity ward nurses faced an emergency, the OPD clinician was the first to see the woman. The OPD also was responsible for triaging incoming patients and determining whether they should 
be admitted to the maternity ward, the gynecology ward, or elsewhere. At this time, as part of triage, the OPD personnel would write a preliminary diagnosis and differentials and would order lab tests and any medications or procedures they thought necessary. Therefore, to understand delays and their part in the process, I always felt the OPD doctor in charge would have added a great deal to the conversations during the audit meetings. Likewise, laboratory personnel were important because they were responsible for ensuring that blood was available for emergency transfusions. The lab was also responsible for confirming any of the diagnoses put forth, most notably malaria in pregnancy or infections. Without inclusion of representatives from all of the departments with which maternity worked, it was difficult to adequately address any delays or gaps in care that had occurred at the hospital. Without including the OPD, it would be impossible to improve continuity of care that would ensure that a woman in critical condition did not become lost in the shuffle of busy wards. The essential role of the OPD is also clear in the description of my quest to locate information related to the two women who had died at the hospital but for whom there were no records. The OPD doctors never had representation and never formally heard the outcomes from these meetings, whether because no one believed them to have a place in the conversations or simply because their inclusion was not part of the meeting routine.

The infrequency of meetings meant that, in addition to more thorough consideration of cases we discussed in the beginning, for the sake of time we did not discuss the oldest cases at all. Those present also tried to make a case for one or more of the deaths not "really" being a maternal death so we that we would not have to spend time going over the details. They might try to suggest this if it appeared that the woman had had preexisting or underlying health problems. Physiologically, nearly any preexisting health problem can be exacerbated by the increased demands that pregnancy places on all of a woman's bodily systems. ${ }^{20}$ For example, to accommodate the fetus, a woman has expanded blood volume throughout the pregnancy, which can put extra strain on her heart or lungs. Deciding to exclude a pregnant woman's death from the data on maternal deaths from the hospital was as much strategic as it was born of a genuine belief that her death had not been caused by her pregnancy. In other cases, the determination to exclude a woman from the count of maternal deaths could be a result of inadequate knowledge of pathology and the complex physiological effects of pregnancy. During these meetings, until the last one I attended in May 2015, we did not have a doctor who was specialized in obstetrics and gynecology, and the hospital never had, to my knowledge, a pathologist. The doctors were already too shorthanded to even consider taking on the additional work of postmortem examinations of women who had died from pregnancy-related causes. Once again, the system itself prohibited some of the very processes that could have helped the hospital gain access to the additional information necessary for improving care. 


\section{CASE FILES}

While the physician in charge of maternity was responsible for calling the meeting, it was his nursing counterpart who was responsible for maintaining the paperwork and preserving the medical records of the women who had died. In theory, the maternity ward nurse in charge was to keep the files of the dead women together in one place, maintaining them until such a meeting happened. However, before almost every meeting there was a panic as the nurse in charge came to realize that one or more case files had gone missing. In these instances, differently than in the case of missing partographs, there were rarely any accusations about foul play involved in these disappearances. Instead, the disappearances were due to poor organization combined with poor communication and a lack of standard procedures for the storage of the files. Inevitably, one or more files would have made their way back to the Medical Records Department or would have been lost in a handover that was never completed.

In practice, the files were never allowed to leave the hospital grounds, but it was not at all uncommon to be unable to find a patient's records. Sometimes doctors borrowed the files to do a more thorough case review or to try to puzzle out why a particularly difficult case had "defeated" them. Once, in 2014, during a period of handover between the incoming and outgoing nurses in charge, we discovered that the files meant for the maternal death audit meeting were locked in the ward storeroom. Only the outgoing nurse in charge possessed the keys, and she had traveled out of the district. Other times, the medical attendant was responsible for opening a patient file after the woman had already died, carrying the papers, which might have been stapled together (or not), to Medical Records. She was then supposed to return to the maternity ward with the new file, its loose papers now tidily constrained, held within the cardstock covers with staples or piece of string onto which hole-punched pages were strung. It was more than possible that some files never made it back to the maternity ward after this detour to Medical Records. Alternatively, they may have made it back to the ward but then been subsequently misplaced when no one knew why a file had been left lying around. Nurses frequently "filed" miscellaneous paperwork of uncertain origin or uncertain trajectory in various cupboards, cabinets, drawers, and boxes.

This "filing system" on the maternity ward was infamous, and during a meeting early in my field stay, Patron called out the maternity ward for putting discharged patients' files in boxes in the corner of the room, in what had yet to become the maternity ward operating theater. He said, "The files have been there for more than a month and have not been taken to Records! The patients have been discharged, but the files are still there! What is this system there on your ward?" But though the system did not follow the hospital protocols, it was a system; the afterlives of these "misfiled" files had important effects on subsequent events, such as the maternal death audit meetings. Kinaya, the nurse in charge at the time of 
Patron's reprimand, went straight back to the ward and instructed the nurses on duty to take files to the Medical Records Department as soon as the patient left. Kinaya muttered under her breath, "I'm talking, talking, but nothing changes," after Nurse Happy listlessly acknowledged this new duty, leaving little certainty that files would actually begin making their way to the records building.

These files were, themselves, characterized by missing information-the wrong times (or none at all) or dates, written hastily while doctors buzzed from one patient to the next on ward rounds. Incomplete medical histories neglected to include details of previous pregnancies and their outcomes. Scrawled doctors' notes in at least three different handwritings wove a carpet of barely legible English instructions, differential diagnoses, and observations on the patient's condition. In every audit meeting we had a discussion about the quality of medical histories, and either the ward doctor in charge or the medical officer in charge implored everyone present to improve intake interviews and timekeeping, which they often portrayed as systemic problems preventing the hospital from further improving care. Here, between the cardboard covers, upon which the woman's name was often misspelled at the whim of the Medical Records personnel, was brought into being a woman in critical condition - a life, or death, hanging together or falling apart on the pages. Missing information made it nearly impossible to reconstruct her pathway to and through the maternity ward.

\section{EXTRAPOLATING WOMEN'S LIVES}

Against this backdrop of uncertainty and barely contained file chaos the actual meetings took place. Because of the length of time that had often passed between a woman's death and the review of her case, the files were all that the meeting attendees had when assessing the progress of the woman's clinical condition, overall health, decision-making skills, family dynamics, and reception and treatment at the hospital. The meeting attendees extrapolated missing information on the basis of where the woman was from, how many previous pregnancies she had had, or the state in which she had arrived at the hospital. For example, the antenatal clinic cards and several of the hospital forms, such as the doctor's notes page, included a line at the top for the patient's religion. The woman's religion was also one of the blanks that needed to be filled on the Ministry of Health forms. Often, the woman's antenatal card did not indicate her religion, and the meeting attendees would infer it on the basis of her name or that of her husband: for example, Asha, married to Mohamed, was almost certainly a Muslim, whereas Anna was more likely to be a Christian. Though these interpretations were based primarily on stereotypes and generalizations about Christians, Muslims, and particular ethnic groups, the meeting participants accepted them in order to fill in the required blanks on the form.

In addition to the uncertainties about such missing individual attributes or demographic information, there was often some uncertainty about how the 
woman's obstetric emergency had unfolded. If the details of her treatment within the hospital were certain, those events that had preceded her arrival often were not clear, based only on what had been gleaned from relatives or the woman herself before her condition had taken a turn for the worse. Occasionally, the woman had arrived at the regional hospital accompanied by a referral letter and maybe even a representative from the referring health facility. In these cases, more information about what had occurred before arrival at the hospital was available. But many times the person accompanying the woman knew little about what had transpired or, even if a health provider, did not have many details about the woman's condition. This was sometimes because of the brief period of the woman's stay at the referring facility or, in other cases, because of a lack of skills and knowledge on the part of a referring provider. If providers did not have training in the recognition and treatment of obstetric emergencies, they might not even have been able to identify the exact complication beyond the fact the woman was bleeding, for example, or that she had been unable to give birth after a prolonged period of time.

In other instances, the woman arrived at the hospital perfectly healthy and her death came as a sudden and unexpected shock even to the providers involved in her case. While no one ever expected a woman to die, some women arrived at the hospital in obviously poor health, and their decline and subsequent death were consequently less of a surprise to the ward staff members. In one particularly bad week in March 2014, just one month after I had arrived at the hospital, several women died within the span of a few days. The most shocking of these deaths was that of Paulina, whose story opens the book. She had reported to the hospital for a scheduled C-section before her labor started. She was in her midtwenties and had had her previous two children via C-section, which was a standard indicator for another surgical delivery. With increased scar tissue in the abdomen and multiple previous incisions in the uterus, there is a greater risk of uterine rupture if the woman experiences strong contractions during labor. In this case, Paulina had arrived early with plenty of time to prepare and schedule her surgery.

The next day, Nurse Lucy, who had been assisting in the operating theater and had gone to receive the baby when it was born, reported back to us on the ward that Paulina had died. She had needed a blood transfusion, but she was blood type O negative, a rare type compatible only with other $\mathrm{O}$ negative blood. There was not enough blood available at the hospital; Paulina received only one unit of blood. In later discussions, the doctors and nurses agreed that her death was likely due to hypovolemic shock from her prolonged internal bleeding. The nurses were dazed, and in the days afterwards Dr. Deo, who had seen Paulina from the time of her arrival at the hospital, was adamant about implementing changes to procedure to prevent similar deaths in the future. On rounds with Dr. Deo almost three weeks after Paulina's death, he told me, "Now, for all cases of planned, non-emergency Caesars, we clinicians must make sure we have the results of the patient's blood type, cross-matching, and hemoglobin tests. We should have two units of blood on 
standby for them, too, before even starting the surgery. We must learn from these other cases and not repeat that mistake!"

To my surprise, when we later discussed Paulina's case in the audit meeting, there was no mention of this proposed protocol, nor did the meeting participants, including Dr. Deo, put this in the action plan. It appeared that once a death lost its immediacy, the nurses, doctors, and administrators quickly returned to the status quo necessitated by their environment. In contrast to how the everyday ethical negotiations in the last chapter created lasting informal structures for accountability, in this instance Dr. Deo's ethical negotiations in the aftermath about what should have been done to prevent Paulina's death did not result in any lasting change. Though he reflected on the events and sought to improve outcomes in the future should a similar case occur, the effects of Dr. Deo's everyday ethics, as individually driven as they were, faded along with the immediacy of Paulina's death.

There was little visible follow-up to address the changes that might have reduced future deaths from similar causes. In some cases, the doctors explained to me, this was because they had started trying to initiate a change or had requested a missing supply but were swiftly met with barrier after barrier or the reluctance of their colleagues and had soon given up the plan. The hospital's Quality Improvement Team (QIT) should also have participated in organizing onthe-job training to improve skills, as well as following up on the requisitioning of needed supplies, such as resuscitation equipment. However, during the majority of my time on the ward the QIT was more of an idea than a functioning body. No one was 100 percent positive about who the maternity ward representatives were, and it was unclear whether they were actually meeting and/or implementing any activities. This lack of certainty about who was responsible for following up or for implementing new protocols and guidelines compounded my feeling that the paperwork resulting from maternal death audit meetings was lost in an unknowable bureaucratic quagmire.

The amount of bureaucracy constructed, at both a national and a local level, around systematically measuring maternal deaths, accounting for them, and implementing programs to reduce these deaths would lead one to believe that the outcomes of such efforts would be consistent and replicable declines in pregnancy-related deaths. After all, dependable replicability is, or was at one time, the objective of bureaucracy-systematization for replicable, predictable, and efficient outcomes, ${ }^{21}$ part of rendering this problem technical. However, the outcomes were more often arbitrary, underanalyzed, and lacking causal certainty; ${ }^{22}$ perhaps declines in maternal death were due to actions on the part of the health care providers and administrators, or, just as likely, any declines were simply a chance occurrence whose continuance into the future was not something that a facility, a region, or the country could depend upon. 
TABLE 1 Example of an action plan

\begin{tabular}{lllll}
\hline Problem & Solution & Person Responsible & Timeline & $\begin{array}{l}\text { Outcome } \\
\text { Indicator }\end{array}$ \\
\hline $\begin{array}{l}\text { No working radio } \\
\text { call, so late referral }\end{array}$ & Fix radio call & $\begin{array}{l}\text { District medical } \\
\text { officer }\end{array}$ & $\begin{array}{l}\text { Within six } \\
\text { months }\end{array}$ & $\begin{array}{l}\text { Will be able to } \\
\text { call for ambulance } \\
\text { with working } \\
\text { radio call system }\end{array}$ \\
\hline
\end{tabular}

\section{THE FUTILITY OF ACTION (PLANS)}

The outcome of the maternal death audit meeting was supposed to be action plans decided upon by the meeting participants. The underlying premise, once again, was that these action plans could structure next steps within the hospital and at the district level that would prevent recurrence of maternal deaths from the same causes. For example, if a woman was delayed in arriving at the regional hospital because the staff at a referring facility needed to call an ambulance from the district but did not have a working radio call system, the action plan might look something like the one shown in table 1.

Sometimes the woman's death could have been prevented by the presence of a specific supply or something as easily remedied-for example, perhaps there had been no adult resuscitation equipment in the operating theater or anyone with the knowledge of how to prevent the woman on the table from aspirating into her lungs secretions that she started to produce during the surgery, as was the case with Kinakia in chapter 2. Kinakia had died, meeting participants speculated, from this aspiration of fluids. This was a relatively straightforward problem with an equally straightforward solution-make sure the resuscitation equipment was present and in good working order and ensure the presence of adequately trained surgical nurses or others versed in recognizing the signs that would necessitate intubation, suction, or other forms of resuscitation.

More often than not, however, the problems the meeting participants decided to include in the action plan boxes on the last page of the Ministry of Health's audit form were not so concrete and self-contained. The needs the meeting participants identified often went along the lines of "better education of pregnant women during the antenatal clinic," and the corresponding outcome indicator was left as "a decrease in maternal deaths." Not only was it impossible to measure the success of prenatal education in this way, but the plan did not actually delineate specific steps to be taken in order to reach "better education" or identify any intermediate indicators that could demonstrate that the plan was on the right track, or give any indication of where the resources to accomplish these plans were supposed to come from within already tight budgets. This method of creating action plans that the region was incapable of executing mainly served the administrators who would be in the responsible position. With little to no time dedicated to actually producing 
steps that would lead to accomplishing the action item, and in the absence of the extra staffing with which to execute them effectively, the administrators sitting around the table at the maternal death audit meeting worked within national reporting structures to fulfill their bureaucratic duties while also ensuring they did not generate more work for themselves. This simplification of maternal deaths that reduced them to impossible action plans was yet another example of the way technical solutions to complex problems, and the fetishization of indicators, precluded actual solutions while all the actual contributing factors did not disappear. ${ }^{23}$

On-the-job training was another popular action item intended to increase providers' skill levels or knowledge of particular procedures, conditions, or interventions. Most administrators believed that any sort of long meeting or training required, at the very least, food for the participants to incentivize attendance. In a region and a hospital that was financially precarious, the prospect of having to provide food for thirty or more maternity ward staff members in order to conduct a training severely limited the possibility of in-house trainings without additional support from NGOs or the Ministry of Health. In a typical example, during the first week of March in 2014, the nurse in charge, Kinaya, organized on-the-job training for the nurses on the ward, to which they had all agreed in a ward meeting a week prior. She intended for them to all walk through the basic emergency obstetric and neonatal care assessment guidelines together, from start to finish, over the course of three days that week. On the first day, nearly two-thirds of the ward nurses were present. The following day, only three people not scheduled to be working the morning shift were present for the instruction. Upon seeing the poor turnout, Kinaya clucked and shook her head disparagingly, saying, "Yaani, humans, for real, it is hard to change their behaviors!" And that was the last time I ever saw or heard any concerted attempt to hold organized, ward-wide, on-the-job training. Additionally, there were no consequences if a maternity staff member did not attend meetings. Again, while Kinaya knew the professionally ethical route was to train her staff members to conduct high-quality care that complied with guidelines, her work environment meant her subordinates did not see the benefit of sacrificing personal time for training on protocols that they could only partially implement because of shortages and high patient loads. Therefore, for all parties involved, it seemed more beneficial, and more ethically sound, to drop the ruse of on-the-job trainings and proceed with actual care work.

If we assume that district health administrators did implement the action plans, in one way or another, in their districts after the audit meetings, they would have been the only ones who knew what they had done or the outcomes of said activities. Because of the infrequency of the audit meetings, and because of time constraints when these did occur, there were so many cases to discuss that the maternity ward medical officer in charge did not dedicate any time to reviewing steps taken since the previous meeting. Never once did I hear a report on whether the gathered administrators had made progress toward accomplishing the action 
plans created in a prior meeting, nor did I ever hear any reports on the stated outcome indicators. The action plans primarily fulfilled reporting requirements and were treated largely as rhetorical documents, as opposed to plans with the real potential to generate change in the system and prevent deaths. If the responsible parties were making progress on the action plan tasks assigned to them, reporting back to the gathered group could have helped create a sense of momentum and possibility, demonstrating that actions did, in fact, produce tangible results. ${ }^{24}$

After the meeting participants completed their action plans, the data traveled only upward. The nurses on the ward, save the few present, who were responsible for everyday care and prevention of these deaths never received a complete report on the meeting's findings. The lack of feedback and communication about action plans, progress made, or interventions planned amplified the sense that any change in the number of maternal deaths was simply a random occurrence. Perhaps it was luck. Perhaps it was due to different staff members. Perhaps it was due to the weather in the region, as when bad rains caused the roads to deteriorate and delayed a woman's arrival even further.

Dr. Charles, while in charge of the maternity ward, and Dr. Joseph, as the medical officer in charge of the hospital, told me I was the only person in the entire hospital who actually used any of the data that the hospital collected to try to draw attention to trends within the institution. I repeatedly suggested that the ward try to use the data that they collected, and that they had to use for reports every month, to help them set goals for care on a quarterly or yearly basis. I has gotten this idea from the maternity nurse in charge whom I had worked with in the Singida region; he had had great success in reducing the incidence of intrapartum stillbirths on his ward through a committed tracking of data. He used the data to show his staff trends in the number of these stillbirths. At Mawingu, instead of using these data, which were being produced to meet the demands and reporting requirements of the central government, as well as NGOs and multilateral programs, for their own ends, the hospital logged these data in the officially required books and rarely looked at them again once the bureaucratic needs were fulfilled.

\section{PERFORMING MATERNAL DEATH AUDITS}

Maternal death audit meetings are another tool, much like the partograph, that people outside Tanzania have constructed and that is meant to aid providers and administrators as they work to continue reducing maternal deaths in their settings. And yet, much like the partograph, the audit meetings rarely took the ideal form they were meant to have. As the smells of the waiting rice, chicken, and chapati from the caterer wafted over to the meeting table, the participants rushed through the details of cases, attempting to rule out a death as related to or caused by pregnancy and simply advising each other to copy the details of the action plan from previously discussed cases. The administrators, nurses, and doctors sitting around 
the table seemed to be engaging in a performativity of the meeting as bureaucratic requirement, as opposed to a tool for changing their ways of practicing care and serving pregnant mothers. The forms and numbers they produced traveled to higher levels and there turned into other numbers, indicators. As Claire Wendland has commented, an indicator "looks like a number and works like a fact, but it is more like the moral of a story" - one that hides uncertainty, highlighting some contributing factors while strategically deemphasizing others. ${ }^{25}$

Overall, while the Rukwa region was, on paper, meeting the demands of the Ministry of Health, and other organizations, for surveillance and reporting of maternal deaths, the fulfilled bureaucratic requirements belie the unsuitability of the maternal death audit system. It is clear that death audits as reproductive governance are not totalizing and do not straightforwardly produce new subjects; instead, "apparently disciplining regimes," here those of maternal health indicators and global safe motherhood, "retain resilience, unpredictability and are shaped by effects of local and biological specifics." ${ }^{26}$ To what extent have tools of governance like the maternal death audit meeting shaped new subjectivities, or in what ways have the simplifications inherent in this globally mobile exercise rooted in public health left open a space for health care workers and administrators to resist their (re)formation as self-disciplining subjects in this global complex around ensuring safe motherhood? ${ }^{27}$ Staff do not actively want to resist the reduction of maternal deaths in their setting, but the forms and biobureaucratic processes to which they are subjected ignore the locally specific spaces in which complex practices of technical and affective care, informal accountability, and clinical improvisation grow and are utterly unable to capture them. What this process reveals are the contributors to maternal death already well known and amenable to technical solutions, proving, perhaps, that the audits work perfectly. ${ }^{28}$ Likewise, the expectations inherent in the forms and rhetorical processes of the audit elide the region's pervasive lack of resources, which is one of the key contributors to the failure of so many potential and attempted interventions. Perhaps the administrators' resistance to the reproductive governance trained on them takes the form of engaging in a performance of the audit meeting that rarely goes deeper than that because they are aware that the expected action plans following a narrow script are unsuited to, and often impossible in, their setting. What this means is that every subsequent meeting will see the emergence of the same barriers, the same challenges, gaps, and pitfalls as have repeatedly been identified in the past. Those problems amenable to technical interventions will make their way into action plans while the deeper roots of maternal mortality, both in the hospital and in the community, will remain unacknowledged and unaddressed. Inequity and resource scarcity remain tenaciously entrenched, escaping technical rendering in the audit process. 